\title{
A computational framework for modelling inter-group behaviour using psychological theory
}

\author{
Rachel Bellamy ${ }^{a}$, Gualtiero Colombo ${ }^{b}$, Soheil Eshghi ${ }^{e},{\text { Geeth De } \mathrm{Mel}^{c} \text {, Cheryl Giammanco }}^{d}$, \\ Rhodri Morris $^{b}$, David G. Rand ${ }^{e},{\text { Liam } \text { Turner }^{b} \text {, Roger M. Whitaker }}^{b}$, Grace-Rose Williams ${ }^{f}$ \\ ${ }^{a}$ IBM T.J. Watson Research Center, 1101 Kitchawan Road, Yorktown Heights, NY, USA 10598 \\ ${ }^{b}$ School of Computer Science \& Informatics, Cardiff University, Cardiff, UK CF24 3AA \\ ${ }^{c}$ Hartree Centre, IBM Research., Warrington, UK WA4 4AD \\ ${ }^{d}$ U.S. Army Research Laboratory, Human Research and Engineering Directorate, Aberdeen \\ Proving Ground, MD, USA \\ ${ }^{e}$ Yale Institute for Network Science, Yale University, New Haven, USA 74085 \\ ${ }^{f}$ Defence Science \& Technology Laboratory, Porton Down, Salisbury, Wiltshire, UK SP4 0JQ
}

\begin{abstract}
Psychological theories of inter-group behaviour offer justified representations for interaction, influence, and motivation for coalescence. Agent-based modelling of this behaviour, using evolutionary approaches, further provides a powerful tool to examine the implications of these theories in a dynamic context. In particular, this can enhance our understanding of the escalation of hostility and warfare, and its mitigation, contributing to policy and interventions. In this paper we propose a framework through which social psychology can be embedded in computation for the examination of inter-group behaviour. We examine how various social-psychological theories can be embedded in evolutionary models, and identify ways in which visualisation can support the objective assessment of emergent behaviour. We also discuss how real-world data can be used to parameterise scenarios on which modelling is conducted.
\end{abstract}

Keywords: group modelling, psychology, evolutionary behaviour, in-group, out-group

\section{INTRODUCTION}

Humans have evolved with the ability to coordinate through groups, which provide a survival advantage. ${ }^{1,2}$ In environments characterised by scarce resources, success from working together against threats has promoted selection in favour of those groups with strong in-group loyalty and cooperation, and strong out-group combativeness against rivals. ${ }^{3,4}$ Aspects of this behaviour are well seen in today's society, reflecting conflict situations on the one hand through to extraordinary acts of altruism and in-group support on the other. Cultural evolution, through the ability to socially interact and copy others plays an important role in this coordination..$^{5,6}$

Understanding the dynamics that underpin behaviour and interactions remains an important area of study that spans a range of disciplines, with potentially diverse methodological approaches. In this paper we are particularly interested in exploring the extent to which computation can be used to increase understanding of how group level interactions occur, and lead to the fracture and formation of groups. This is particular relevant to the importance of groups in threats to world peace. While previously the cold war and its associated arms race was characterised by clearly identifiable actors, formal administrations, territorial boundaries and observable behaviour, ${ }^{7}$ many modern conflicts are less clear cut and much more challenging to explicitly model and understand. $^{8}$ Groups play a particularly important role in this context, but rationally modelling and understanding group behaviour and conflict is a challenge, particularly in extreme situations.

One approach to making progress is through computational modelling. Generally speaking, the advantage of this methodological approach is that scenarios can be developed and assumptions tested that allow a deeper understanding of complex dynamics to be ascertained. Users may rerun and interpret many different and

Send correspondence to R.M. Whitaker, Email: whitakerrm@cardiff.ac.uk, Tel: +44 (0) 2920876999 
hypothetical situations that allow insights to be built. It is also possible to address large-scale scenarios and emulate thousands of interactions that are difficult to observe by any other means. However the complexity of human behaviour necessitates some form of abstraction takes place, because it is impossible to model or even represent every facet of human behaviour in a computational or quantitative form.

In this paper we argue that progress can be made however, by basing computational models on established theory, or testable assumptions, that represent different aspects of human psychological behaviour. This approach has a number of important aspects. Firstly it means that due diligence has been paid, through development of the theory itself, to actions and consequences of human behaviour. Secondly it allows static theory, which has been developed on the observation individuals or small samples, to be examined in a dynamic setting, such as when many individuals interact based on specific assumptions about each response behaviour. Thirdly it allows dynamics from assumptions about individual behaviour to be better understood, in terms of the characteristics that groups represent.

However behaviour that can be represented through computation requires theory that can be readily detected and discretised into actions. Qualitative theory in particular is therefore challenging to translate, but theories based on interaction or representation through functions lend themselves more in this direction. We present examples of such theories and approaches from psychology, also giving insights concerning their implementation in a wider event-driven framework. This is focussed on in-group and out-group effects, which represent a fundamental starting point for modelling inter-group behaviour.

\section{SNAPSHOT OF KEY THEORY CONCERNING INTER-GROUP BEHAVIOUR}

The origins of relevant theory date back to the turn of twentieth century. Early psychological contributions on structural conflict theory ${ }^{9}$ and realistic conflict theory ${ }^{10,11}$ were aligned with environments characterised by promoted selection in favour of those groups with strong in-group loyalty and cooperation, and strong out-group combativeness against rivals. However these early contributions fell short of explaining why biased group-based attitudes may persist even in the absence of conflict or resource scarcity. Allport ${ }^{12}$ proposed that susceptibility to group-based stereotyping ${ }^{13}$ was a contributory factor, being an efficient heuristic that avoids deliberation but leaves social judgements susceptible to erroneous influences and over-generalisation in respect of group members. ${ }^{14}$

Allport's Intergroup Contact Hypothesis ${ }^{12}$ proposed that positive effects of intergroup contact occur only when there is equal group status, common goals, intergroup cooperation and the support of authorities, law or custom. Intergroup contact theory ${ }^{15,16}$ has subsequently extended these conditions and sought to explain processes by which contact changes attitudes and behaviour, specifically through learning about the out-group, changing behaviour, generating effective ties and reappraising the in-group. This theory predicts that patterns of contact between groups can counteract over-generalisation and mitigate prejudice when members of respective groups can identify with each other.

More generally, social identity has emerged as the predominant paradigm for understanding intergroup phenomena. ${ }^{17-19}$ Self-identification of the in-group based on a common identity, ${ }^{20}$ whether based on fixed traits (e.g., ethnicity or religion) or mutable beliefs (e.g., opinions or preferences), has been shown to predict in-group favoritism as well as out-group hostility. ${ }^{18,21,22}$ Much of the research in this area has sought to understand the division between the in-group and out-group. Given the correspondence between the definitions of an in-group and out-group (i.e., one implies the other), in-group bias is often conflated with out-group prejudice, ${ }^{23}$ resulting in an implicit and erroneous assumption that these concepts are interchangeable. Therefore the considerable research on the behaviour and conditions that support in-group favoritism ${ }^{19,24-26}$ is insufficient to determine the conditions that promote prejudicial attitudes. Current knowledge of the processes that support the in-group reinforcement are framed through advanced cognition, such as affective capabilities, social intelligence and intuition over deliberation. ${ }^{13,14,27}$

More recently, there has also been theorising on the role of an individual's identity in terms of group behaviour. The concept of identity fusion ${ }^{28}$ has been developed, under which conditions are such that individual identity is lost and substituted for group identity. This is a contributory factor in understanding how individuals become compliant with extreme behaviours that are a function of the group ${ }^{29}$ and represent "unconditional commitment 
and intractability" ${ }^{30}$ Collectively these contributions represent influential aspects of the literature, describing ways in which individuals and groups could interact. The challenge comes in engaging these theoretical concepts in a computational form.

\section{EMPLOYING COMPUTATION}

There are diverse approaches to modelling group behaviour in a computational form. Different approaches represent a continuum between modelling the interactions of individual actors independent of groups, as opposed to recognising a group as an entity in its own right with characteristics capable of influence or action on others. ${ }^{31}$ There are numerous approaches, at a high level, that seek to make deductions based on structure and interaction rather than explicit psychological theories. Examples include the biologically inspired "Blau space", ${ }^{32}$ which functions based on homophily, - that is those with similar socio-demographic characteristics are more likely to be associated by involvement in similar groups. Blau spaces project this information and find equilibrium between potentially competing groups, using a system of differential equations to derive a competition matrix between finite resources (individual participation). Explicitly modelling systems dynamics is also possible. This uses nonlinear relationships between the forces influencing group behaviour, ${ }^{33}$ employing "stocks and flows" which are the abstract representations of virtual (or physical) commodities and their local connection and influence. Hybrid approaches to modelling have also emerged ${ }^{34,35}$ which combine multiple layers of cognitive modelling with a wide range of variables. However, to make progress on modelling while taking into account explicit psychological theory, it is reasonable to focus on representing individuals within the model. Agent-based modelling is a particularly useful vehicle to achieve this, and defines the scope of our interest in this paper.

\subsection{Understanding in-group and out-group effects: a framework}

In light of considerable previous work, we argue that a key point of reference for understanding inter-group behaviour concerns in-group and out-group effects, and therefore focussing attention on this is important. Conflict is frequently group-based and understanding how social and psychological dynamics support positive in-group behaviour and negative out-group behaviour is highly valuable. Bias and inter-group conflict remain a "problem of the century", ${ }^{36}$ and frame many of society's divisions.

In-group assortment is well-seen in human behaviour, being easily triggered by attraction on the basis of some degree of similarity. Lab-based experiments have shown that this can occur based on trivial or arbitrary displays of similarity. ${ }^{6,22,24,37}$ This has contributed to a misperception that positive discrimination to the ingroup and negative discrimination to the out-group are inevitable. ${ }^{23}$ Consequently understanding the separate roles of in-group and out-group discrimination is socially important, in particular understanding how it becomes manifested through social mechanisms. Psychological theory can be embedded in different aspects of agentbased computational simulation, ${ }^{38}$ and is well-suited to exploring in-group and out-group effects. We focus on evolutionary modelling, where natural selection, after periods of interaction, is repeatedly applied. Psychological and social considerations can be embedded in such models and their effects observed. ${ }^{39}$ In particular we highlight four key considerations where theory can be embedded to further understand group dynamics.

- Scope of interactions. This issue relates to how individuals are assumed to mix and interact. This can be affected by a-priori levels of discrimination, either positive towards the in-group, negative towards towards the out-group. Equally observation of the evolution of these factors may be the purpose. Modelling decisions can be informed by potential scenarios and there are a range of examples in the literature. For example, a-priori structure from placing agents on a regular lattice has been previously adopted ${ }^{26}$ using the lattice to control the possible interactions with only adjacent agents. Rewiring of this lattice has also been considered, ${ }^{40}$ allowing the co-evolution of interactions alongside other agent-based behaviour. Other approaches have involved sampling agents on a probabilistic basis from sets (i.e., groups), and allowing agents to freely change sets. ${ }^{25}$ Segregation of the population into islands is also possible. ${ }^{41}$

- Form of interactions. Interactions need to be characterised in terms of actions and responses. Social and psychological theories can be embedded at this point, ranging from assumptions about mimicry, social learning ${ }^{42}$ or attraction through homophily. ${ }^{25}$ Game theory is often (but not always) used in this context, 
being a mechanism through which individuals are faced with a social dilemma. Decision-making in this context occurs as a consequence of a social dilemma - where agents are faced with an interaction through which cooperation may occur. ${ }^{43}$ Wide-ranging social, psychological and economic factors can have influence. For example, these may involve the social norms of a group, ${ }^{44}$ strategies being inherited from others in the population, such as through cultural means, ${ }^{42}$ as a consequence of identity, ${ }^{28}$ or reinforcement of decisions from multiple sources. Social Impact Theory, proposed by Latané ${ }^{45}$ is one example of how an individual may be influenced by multiple factors, as explored in contexts such as opinion formation, beliefs and culture. ${ }^{46,47}$

- Basis for discrimination. Within modelling, agents need to be able to identify ${ }^{48}$ with others, individually or collectively, to determine the extent that they are in-group or out-group. The agent's disposition towards discrimination represents their attitude, framing an individuals world view. Humans are well-known to act on the basis of social comparison ${ }^{49,50}$ and this has been found to be important for cooperation scenarios based on donation. ${ }^{51}$ Various approaches to assessing similarity have been proposed. For example, may agents carry a number of discrete traits may compare themselves for similarity, with actions proportional to the extent of commonality. ${ }^{42}$ More generally, a range of tag-based models ${ }^{26,52-58}$ are known, that are used to spontaneously enable cooperation based on propagation of behavioural strategies. Social norms of the group may also be influential over group members' discriminatory behaviour, ${ }^{59}$ and the role of social norms in cooperation is significant ${ }^{44}$ in potentially supporting punishment. ${ }^{60}$ Discrimination can also take the form of reputation distortion, preferential selection for interaction, or particular actions, such as out-group hostility concurrently with in-group favoritism..$^{3,4,61-63}$

- Consequences of interaction. As the result of behaviour, individuals and groups are affected. This can be modelled in wide-ranging social or economic terms. Exit from a group, either as a consequence of the group (e.g., ostracism ${ }^{64}$ ) or by the individual walking away ${ }^{65}$ is an important consideration. Humans are well-disposed to detecting potential ostracism in a group setting. ${ }^{66}$ Additionally representation of activity in economic terms, through the potential costs and benefits of interaction are commonplace, particularly in game theoretic models that are cooperation based. ${ }^{43}$ Reputation systems ${ }^{67,68}$ are also a further way in which individual behaviours can be assessed, used as a measure of social standing. ${ }^{69}$ These may operate in conjunction with social norms, that are the basis for judgements. Different groups may have different perspectives, as represented by social norms or group identity, leading to different forms of social penalty or reward. This may also take the form of punishment. ${ }^{60}$

\subsection{Visualisation and interpretation}

Finally we remark on the importance of visualisation and interpretation of generative models. Simulations involving large numbers of agents may result in complex feedback to the user which can be challenging to represent and assess. ${ }^{70}$ This is also true with regard to real-world data sets, ${ }^{71}$ and understanding the relationships between visually represented actions and system parameters (e.g., real time social network metrics) in human terms is a step which is critical in aiding consistent 'tellability' and interpretation. Users with different perceptions and experiences may well be susceptible to different types of bias in making judgements on observed changes to a scenario. If it becomes possible to make this connection between the metrics characterising a system and the visual behaviour of that system then prospects to enhance human-machine intelligence will be furthered.

\subsection{Conclusions}

In this paper we have presented insights into how social and psychological theory can be embedded into evolutionary agent-based simulations. There are a range of relevant social and psychological theories that can be incorporated into models. We focus on the importance of in-group and out-group behaviour, and highlight degrees of freedom concerning the incorporation of theory into the model. These choices relate to the scope and form of interactions between agents, the basis for discrimination and the possible consequences of interaction on the individual or group. We also remark on the importance of making further developments with visualisation and interpretation of models of this nature. 


\section{ACKNOWLEDGEMENT}

This research was sponsored by the U.S. Army Research Laboratory and the U.K. Ministry of Defence under Agreement Number W911NF-16-3-0001. The views and conclusions contained in this document are those of the authors and should not be interpreted as representing the official policies, either expressed or implied, of the U.S. Army Research Laboratory, the U.S. Government, the U.K. Ministry of Defence or the U.K. Government. The U.S. and U.K. Governments are authorized to reproduce and distribute reprints for Government purposes notwithstanding any copy-right notation hereon.

\section{REFERENCES}

1. R. Dunbar, Grooming, gossip, and the evolution of language, Harvard University Press, 1998.

2. R. Byrne and A. Whiten, "Machiavellian intelligence: social expertise and the evolution of intellect in monkeys, apes, and humans (oxford science publications)," 1989.

3. H. Rusch, "The evolutionary interplay of intergroup conflict and altruism in humans: a review of parochial altruism theory and prospects for its extension," Proceedings of the Royal Society of London B: Biological Sciences 281(1794), p. 20141539, 2014.

4. J.-K. Choi and S. Bowles, "The coevolution of parochial altruism and war," science 318(5850), pp. 636-640, 2007.

5. S. Bowles and H. Gintis, "Origins of human cooperation," Genetic and Cultural Evolution of Cooperation 2003, pp. 429-43, 2003.

6. C. Efferson, R. Lalive, and E. Fehr, "The coevolution of cultural groups and ingroup favoritism," Science 321(5897), pp. 1844-1849, 2008.

7. L. F. Richardson, Arms and insecurity: A mathematical study of the causes and origins of war, Boxwood Press, 1960.

8. P. K. Davis and K. Cragin, Social science for counterterrorism: Putting the pieces together, Rand Corporation, 2009.

9. W. G. Sumner, Folkways, Ginn and Company, 1906.

10. M. Sherif and C. W. Sherif, "Groups in harmony and tension; an integration of studies of intergroup relations.," 1953.

11. M. Sherif, In common predicament: Social psychology of intergroup conflict and cooperation, Houghton Mifflin comp, 1966.

12. G. W. Allport, The nature of prejudice, Cambridge/Reading, MA: Addison-Wesley, 1954.

13. P. G. Devine, "Stereotypes and prejudice: Their automatic and controlled components.," Journal of personality and social psychology 56(1), p. 5, 1989.

14. C. M. Judd and B. Park, "Definition and assessment of accuracy in social stereotypes.," Psychological review 100(1), p. 109, 1993.

15. T. F. Pettigrew, "Intergroup contact theory," Annual review of psychology 49(1), pp. 65-85, 1998.

16. T. F. Pettigrew and L. R. Tropp, "Does intergroup contact reduce prejudice? recent meta-analytic findings," Reducing prejudice and discrimination 93, p. 114, 2000.

17. H. Tajfel, "Social psychology of intergroup relations," Annual review of psychology 33(1), pp. 1-39, 1982.

18. M. B. Brewer and R. M. Kramer, "The psychology of intergroup attitudes and behavior," Annual review of psychology 36(1), pp. 219-243, 1985.

19. J. F. Dovidio, S. L. Gaertner, and A. Validzic, "Intergroup bias: status, differentiation, and a common in-group identity.," Journal of personality and social psychology 75(1), p. 109, 1998.

20. S. Reicher, "The determination of collective behaviour," Social identity and intergroup relations , pp. 41-83, 1982.

21. E. Harmon-Jones, J. Greenberg, S. Solomon, L. Simon, et al., "The effects of mortality salience on intergroup bias between minimal groups," European Journal of Social Psychology 26(4), pp. 677-681, 1996.

22. H. Tajfel, M. G. Billig, R. P. Bundy, and C. Flament, "Social categorization and intergroup behaviour," European journal of social psychology 1(2), pp. 149-178, 1971. 
23. M. B. Brewer, "The psychology of prejudice: Ingroup love and outgroup hate?," Journal of social issues 55(3), pp. 429-444, 1999.

24. D. Balliet, J. Wu, and C. K. De Dreu, "Ingroup favoritism in cooperation: A meta-analysis.," 2014.

25. F. Fu, M. A. Nowak, N. A. Christakis, and J. H. Fowler, "The evolution of homophily," Scientific reports 2 , p. 845, 2012.

26. R. A. Hammond and R. Axelrod, "Evolution of contingent altruism when cooperation is expensive," Theoretical population biology 69(3), pp. 333-338, 2006.

27. A. Bear and D. G. Rand, "Intuition, deliberation, and the evolution of cooperation," Proceedings of the National Academy of Sciences 113(4), pp. 936-941, 2016.

28. W. B. Swann Jr, A. Gómez, D. C. Seyle, J. Morales, and C. Huici, "Identity fusion: The interplay of personal and social identities in extreme group behavior.," Journal of personality and social psychology 96(5), p. 995, 2009.

29. H. Sheikh, Á. Gómez, and S. Atran, "Empirical evidence for the devoted actor model," Current Anthropology 57(S13), pp. S204-S209, 2016.

30. S. Atran, "The devoted actor: unconditional commitment and intractable conflict across cultures," Current Anthropology 57(S13), pp. S192-S203, 2016.

31. R. M. Whitaker, D. Felmlee, D. C. Verma, A. Preece, and G.-R. Williams, "From evolution to revolution: understanding mutability in large and disruptive human groups," in Next-Generation Analyst V, 10207, p. 1020703, International Society for Optics and Photonics, 2017.

32. M. McPherson, "An ecology of affiliation," American Sociological Review , pp. 519-532, 1983.

33. N. Choucri, D. Goldsmith, S. Madnick, D. Mistree, J. B. Morrison, and M. Siegel, "Using system dynamics to model and better understand state stability," 2007.

34. M. L. Bernard, G. A. Backus, A. B. Bier, and S. F. Branch, "Behavioral influence assessment (bia): A multi-scale system to assess dynamic behaviors within groups and societies across time," Advances in CrossCultural Decision Making 5, p. 161, 2014.

35. A. B. Bier and M. L. Bernard, "Validating a hybrid cognitive-system dynamics model of team interaction," Advances in Cross-Cultural Decision Making 5, p. 209, 2014.

36. S. T. Fiske, "What we know now about bias and intergroup conflict, the problem of the century," Current Directions in Psychological Science 11(4), pp. 123-128, 2002.

37. T. Yamagishi, N. Jin, and T. Kiyonari, "Bounded generalized reciprocity: Ingroup boasting and ingroup favoritism," Adv Gr Process 16, pp. 161-197, 1999.

38. E. Bonabeau, "Agent-based modeling: Methods and techniques for simulating human systems," Proceedings of the National Academy of Sciences 99(suppl 3), pp. 7280-7287, 2002.

39. J. M. Epstein, "Agent-based computational models and generative social science," Complexity 4(5), pp. 4160,1999 .

40. D. Centola, J. C. Gonzalez-Avella, V. M. Eguiluz, and M. San Miguel, "Homophily, cultural drift, and the co-evolution of cultural groups," Journal of Conflict Resolution 51(6), pp. 905-929, 2007.

41. O. Leimar and P. Hammerstein, "Evolution of cooperation through indirect reciprocity," Proceedings of the Royal Society of London B: Biological Sciences 268(1468), pp. 745-753, 2001.

42. R. Axelrod, "The dissemination of culture: a model with local convergence and global polarization," Journal of conflict resolution 41(2), pp. 203-226, 1997.

43. M. A. Nowak, "Five rules for the evolution of cooperation," science 314(5805), pp. 1560-1563, 2006.

44. E. Fehr, U. Fischbacher, and S. Gächter, "Strong reciprocity, human cooperation, and the enforcement of social norms," Human nature 13(1), pp. 1-25, 2002.

45. B. Latané, "The psychology of social impact.," American psychologist 36(4), p. 343, 1981.

46. A. Nowak, J. Szamrej, and B. Latané, "From private attitude to public opinion: A dynamic theory of social impact.," Psychological Review 97(3), p. 362, 1990.

47. B. Latané, "Dynamic social impact: The creation of culture by communication," Journal of Communication 46(4), pp. 13-25, 1996.

48. M. A. Hogg, "Social identity theory," Contemporary social psychological theories 13, pp. 111-1369, 2006. 
49. A. P. Buunk and F. X. Gibbons, "Social comparison: The end of a theory and the emergence of a field," Organizational Behavior and Human Decision Processes 102(1), pp. 3-21, 2007.

50. L. Festinger, "A theory of social comparison processes," Human relations 7(2), pp. 117-140, 1954.

51. R. M. Whitaker, G. B. Colombo, S. M. Allen, and R. I. Dunbar, "A dominant social comparison heuristic unites alternative mechanisms for the evolution of indirect reciprocity," Scientific Reports 6, 2016.

52. R. L. Riolo, M. D. Cohen, and R. Axelrod, "Evolution of cooperation without reciprocity," Nature 414(6862), p. $441,2001$.

53. G. Roberts and T. N. Sherratt, "Behavioural evolution (communication arising): Does similarity breed cooperation?," Nature 418(6897), p. 499, 2002.

54. D. Hales, "Cooperation without memory or space: Tags, groups and the prisoners dilemma," in International Workshop on Multi-Agent Systems and Agent-Based Simulation, pp. 157-166, Springer, 2000.

55. V. A. Jansen and M. Van Baalen, "Altruism through beard chromodynamics," Nature 440(7084), p. 663, 2006.

56. A. Traulsen and M. A. Nowak, "Chromodynamics of cooperation in finite populations," PLoS One 2(3), p. e270, 2007.

57. N. Masuda and H. Ohtsuki, "Tag-based indirect reciprocity by incomplete social information," Proceedings of the Royal Society of London B: Biological Sciences 274(1610), pp. 689-695, 2007.

58. T. Antal, H. Ohtsuki, J. Wakeley, P. D. Taylor, and M. A. Nowak, "Evolution of cooperation by phenotypic similarity," Proceedings of the National Academy of Sciences 106(21), pp. 8597-8600, 2009.

59. J. Jetten, R. Spears, and A. S. Manstead, "Intergroup norms and intergroup discrimination: Distinctive self-categorization and social identity effects.," Journal of personality and social psychology $\mathbf{7 1}(6)$, p. 1222, 1996.

60. E. Fehr and U. Fischbacher, "Third-party punishment and social norms," Evolution and human behavior 25(2), pp. 63-87, 2004.

61. S. Bowles, "Being human: Conflict: Altruism's midwife," Nature 456(7220), pp. 326-327, 2008.

62. H. Bernhard, U. Fischbacher, and E. Fehr, "Parochial altruism in humans," Nature 442(7105), p. 912, 2006.

63. K. Abbink, J. Brandts, B. Herrmann, and H. Orzen, "Parochial altruism in inter-group conflicts," Economics Letters 117(1), pp. 45-48, 2012.

64. D. Hirshleifer and E. Rasmusen, "Cooperation in a repeated prisoners' dilemma with ostracism," Journal of Economic Behavior \& Organization 12(1), pp. 87-106, 1989.

65. C. A. Aktipis, "Is cooperation viable in mobile organisms? simple walk away rule favors the evolution of cooperation in groups," Evolution and Human Behavior 32(4), pp. 263-276, 2011.

66. N. L. Kerr and J. M. Levine, "The detection of social exclusion: Evolution and beyond.," Group Dynamics: Theory, Research, and Practice 12(1), p. 39, 2008.

67. M. Milinski, "Reputation, a universal currency for human social interactions," Phil. Trans. R. Soc. $B$ 371(1687), p. 20150100, 2016.

68. E. Fehr, "Human behaviour: don't lose your reputation," Nature 432(7016), pp. 449-450, 2004.

69. J. Sabater and C. Sierra, "Review on computational trust and reputation models," Artificial intelligence review 24(1), pp. 33-60, 2005.

70. R. M. Whitaker, L. Turner, G. Colombo, D. Verma, D. Felmlee, and G. Pearson, "Intra-group tension under inter-group conflict: a generative model using group social norms and identity," in International Conference on Applied Human Factors and Ergonomics, pp. 167-179, Springer, 2017.

71. L. D. Turner, G. B. Colombo, R. M. Whitaker, and D. Felmlee, "Parameterising the dynamics of inter-group conflict from real world data," 